\title{
Die Forensische Psychiatrie ist der Medizin und dem Recht verpflichtet
}

\section{Marc Grafa, Elmar Habermeyer ${ }^{b}$}

a Prof. Dr. med., Klinikdirektor und Chefarzt Forensisch-Psychiatrische Klinik, Universitäre Psychiatrische Kliniken (UPK) Basel;

${ }^{b}$ Prof. Dr. med., Direktor Klinik für Forensische Psychiatrie, Psychiatrische Universitätsklinik Zürich

\author{
Die Forensische Psychiatrie befindet sich in einem Spannungsfeld zwischen Patien- \\ ten- und Sicherheitsinteressen. Mario Gmür hat ihr Unwissenschaftlichkeit und \\ unethisches Verhalten vorgeworfen. Der Beitrag widerlegt diesen Vorwurf und \\ verweist auf den medizinischen und kriminologischen Wissensstand.
}

\begin{abstract}
Mario Gmür hat sich in den letzten Jahren in der Laienpresse als Kritiker der Forensischen Psychiatrie hervorgetan. Nun wirft er der Forensischen Psychiatrie in der SÄZ 36/2014 [1] ${ }^{*}$ vor, sich vor dem Hintergrund eines durch mediale Berichterstattungen geschürten übersteigerten Sicherheitsbedürfnisses der Gesellschaft zum Steigbügelhalter eines inhumanen Strafund Massnahmevollzugs zu machen, mit Prognoseinstrumenten "Wissenschaftsbetrug» zu betreiben, «sittenwidrig» Psychotherapie "punitiv» einzusetzen, und er fordert «ein disziplinarisches und strafrechtliches Regelwerk» "gegen die Missachtung ethischer Normen durch das therapeutische Personal». Eine solche pauschalisierende Generalabrechnung mit unserem Fachgebiet kann nicht unwidersprochen bleiben.
\end{abstract} www.saez.ch $\rightarrow$ Aktuelle Ausgabe oder $\rightarrow$ Archiv $\rightarrow 2015 \rightarrow 12$

\section{Aufgaben der Forensischen Psychiatrie}

Die Forensische Psychiatrie stellt sicher, dass psychisch kranke Straftäter entsprechend ihrem Recht auf eine fachlich korrekte und menschenwürdige Therapie behandelt und gleichzeitig Dritte möglichst vor Straftaten durch psychisch kranke Menschen geschützt werden. Noch mehr als die Psychiatrie im Allgemeinen muss sich die Forensische Psychiatrie wegen des hohen Missbrauchspotentials selbst reflektieren und fachliche, ethische sowie rechtliche Standards laufend überprüfen. In der Forensischen Psychiatrie wird das Spannungsfeld zwischen Patienten- und Sicherheitsinteressen zwar am deutlichsten sichtbar, es unterscheidet sich aber qualitativ nicht von der Situation in der medizinischen Praxis, wenn z.B. zwischen einer Meldung an die Kindesschutzbehörde einerseits und der Wahrung des Arztgeheimnisses andererseits abgewogen werden muss. Insofern findet ärztliches Handeln auch ausserhalb der Forensik keinesfalls ausschliesslich in einer dyadischen Beziehung zwischen Patient und Arzt statt.

Der Abgleich zwischen Patienten- bzw. Freiheitsrechten und Sicherheitsinteressen ist eine schmale Gratwanderung: Wo die Gratschneide verläuft, legen die demokratisch gewählten Gesetzgeber und die ebenso legitimierten Gerichte fest. Wer Gerichtsurteile analysiert, wird feststellen, dass die Gerichte dieser Aufgabe mit hoher Sorgfalt und Verantwortung nachkommen. Eine ähnlich hohe Verantwortung tragen forensische Psychiater für täglich Dutzende von Lockerungsentscheiden und die Konsequenzen bei Rückfällen. Wie kann man mit dieser Verantwortung umgehen? Indem man sich immer wieder vor Augen hält, dass die Forensische Psychiatrie alleine der medizinischen Wissenschaft sowie dem Recht verpflichtet ist. Und damit zurück zum Beitrag von Dr. Gmür: 


\section{Vermeintlicher "Wissenschaftsbetrug» im Kontext von Prognose und Therapie}

Mario Gmür kritisiert eine ungenügende Beurteilerübereinstimmung bei Prognoseinstrumenten. Er erhebt diese Vorwürfe, ohne auch nur ein einziges Beispiel für die wissenschaftliche Evidenz seiner These offenzulegen. Dass solche «Argumentationslinien» im offiziellen Organ der FMH publiziert werden, ist peinlich für den wissenschaftlichen Anspruch unseres Fachs. Wir können in der Schweizer Psychiatrie stolz sein, dass schon E. Bleuler dieses unwissenschaftliche "autistisch-undisziplinierte Denken» in einem bereits zu Beginn des letzten Jahrhunderts publizierten und auch heute noch hochaktuellen Werk aufs Schärfste kritisiert hat [2], und sollten uns gegen einen Rückfall in solche Denkmuster wehren. Jedem Leser ist klar, dass in der Forensischen Psychiatrie aus ethischen Überlegungen heraus kaum Doppelblindstudien durchgeführt werden können. Wenn jegliches ärztliches Handeln jenseits von hohem Cochrane-Evidenz-Niveau (randomisierte Doppelblindstudien) wie von Dr. Gmür - pauschal diffamiert wird, ist dies für die Medizin insgesamt gefährlich. Ausserdem zeigen Studien ein gänzlich anderes als das von Dr. Gmür skizzierte Bild.

\section{Von «Wissenschaftsbetrug» kann bei} Anwendung der Instrumente keine Rede sein.

Ausserdem unterliegt er einem fundamentalen Irrtum, wenn er von einer ungenügenden Beurteilerübereinstimmung in «der freien Wildbahn der forensisch-psychiatrischen Gutachtertätigkeit» spricht. Tatsächlich besteht bei in der Anwendung der Prognoseinstrumente geschulten Untersuchern eine ausgesprochen hohe Übereinstimmung: So führt Hare [3] bezüglich der Psychopathy-Checkliste einen Wert von .87 für den Korrelationskoeffizienten in Klassen (ICC) an, von Harris et al. wird für den Static-99, der zur Vorhersage erneuter Sexualdelikte entwickelt worden ist, ebenfalls ein ICC-Wert von .87 berichtet [4]. Für beide Verfahren ergaben sich auch «im Feld» (sprich: bei der Anwendung durch Praktiker ausserhalb von Forschungsprojekten) gute bis sehr gute Kennwerte für die Beurteilerübereinstimmung [z.B. 5, 6]. Diskrepanzen in den Einschätzungen verschiedener Beurteiler treten auf, wenn jene in der Anwendung der entsprechenden Verfahren nicht oder nur unzureichend geschult sind bzw. sich als Parteigutachter als Sachwalter der Interessen nur einer Prozesspartei verstehen [7, 8]. Ein ethisches Problem besteht folglich dann, wenn forensisch-psychiatrische Gutachter solche Instrumente ohne eine Schulung einsetzen bzw. die ihrem Einsatz zugrunde liegenden Manuale nicht kennen. Ein solches Vorgehen kann jedoch nicht der Forensischen Psychiatrie allgemein zum Vorwurf gemacht werden, sondern entspricht einem individuellen Fehlverhalten.

Spätestens seit der Publikation von Grove und Meehl 1996 [9] ist ausserdem belegt, dass strukturierte Beurteilungen in vielen Lebensbereichen, wie Personalrekrutierung, medizinische Diagnostik und Kriminalprognostik, den unstrukturierten deutlich überlegen sind. Auch aktuelle Metaanalysen zeigen diese Überlegenheit in aller Deutlichkeit, zum Beispiel bei der Rückfallprognose für Sexualstraftäter [10]. Von «Wissenschaftsbetrug» kann daher bei Anwendung solcher Instrumente keine Rede sein.

Darüber hinaus hat sich in der Forensischen Psychiatrie die Einbettung der standardisierten Verfahren in ein «structured professional judgement» [11] etabliert, so wie es auch von einer Expertengruppe gefordert [12] und von der Schweizer Gerichtspraxis [13] übernommen wurde. Somit liefern die Prognoseinstrumente nur einen Orientierungsrahmen für die individuelle Einzelfallentscheidung. Der Gutachter soll kein intuitives Urteil abgeben, sondern auf dem wissenschaftlichen Sachstand, und zu diesem gehören nun auch anhand von Prognoseinstrumenten durchgeführte Rückfallstudien, die fachlich fundierte Aussagen treffen, die der Jurist mangels psychiatrisch-kriminologischer Expertise nicht geben kann.

Im zweiten Teil seiner Polemik befasst sich Dr. Gmür mit der vermeintlich repressiven Psychotherapie im Massnahmevollzug. Er verweist dabei, erneut ohne Quellenangabe, auf den Kriminologen Killias, dem zufolge die Überlegenheit von Therapien im Strafvollzug gegenüber Spontanverläufen nicht überzeugend nachgewiesen sei. Diesbezüglich besteht in der Tat ein Forschungsbedarf und insbesondere ein Bedarf nach Studien im deutschsprachigen Raum. Dennoch sei beispielhaft auf die Publikation von Olver [14] verwiesen, die deutlich positive Effekte von deliktorientierten Therapien auf Rückfallfreiheit nachweist. Auch Daten von Lipsey u. Cullen [15] sprechen für die Wirksamkeit psychiatrischer Interventionen, insbesondere der kognitiven Verhaltenstherapie, in Strafvollzugsstichproben.

Weiterhin ist es falsch, wenn Herr Dr. Gmür behauptet, dass für die Behandlung von Persönlichkeitsstörungen keine Methode von durchschlagendem Erfolg bekannt sei. In den letzten Jahren wurden nämlich unterschiedlichste, klinisch integrative Methoden entwickelt, mit denen Persönlichkeitsstörungen mit guten Behandlungsaussichten therapiert werden können $[16,17]$. Umgekehrt besteht keinerlei naiver Thera- 
pieoptimismus mehr: Dass Straftäter mit bestimmten Persönlichkeitsmerkmalen einer solchen Therapie wenig zugänglich sind, resp. diese gar kontraproduktiv wirken kann, ist evident [18-21]. Wie in anderen Bereichen der Medizin werden in der Forensischen Psychiatrie Therapien zur Reduktion der Rückfallraten nach Differentialindikation eingesetzt.

\section{Es gibt nicht nur ethische Verpflichtungen gegenüber dem Patienten, sondern auch gegenüber potentiellen Opfern.}

Wenn Herr Dr. Gmür von «repressiver Psychotherapie» spricht, meint er wohl Zwang zur Therapie, Drohungen, Abwertungen und destruktive Konfrontation. Dies ist kaum zielführend, weder zur Symptomreduktion noch zur Verbesserung der Legalprognose. Weshalb eine solche "ausnahmsweise und für eine beschränkte Zeit in einem Ausmass und Umfang erfolgen [darf], wie sie im Rahmen des Erwachsenenschutzrechts geduldet ist», erschliesst sich uns nicht. Das Erwachsenenschutzrecht dient dem Schutz der von einem Schwächezustand betroffenen Person, Repression ist da wohl nicht angezeigt.

Auch in der Forensischen Psychiatrie sind solche Ansätze seit langem als kontraproduktiv erkannt: Vielmehr werden therapeutische Haltungen gefordert, welche die Bedeutung von Hoffnung und Selbstwertschätzung des Patienten in einer Kooperation mit den Therapeuten fördern [22]. Ärztinnen und Ärzte dürfen und sollen in einem forensischen Kontext Therapien anbieten und versuchen, Patienten dafür zu motivieren (Aufzeigen von Vor- und Nachteilen von Therapie vs. keine Therapie) und die Therapiemotivation aufrechtzuerhalten. Zu den möglichen Nachteilen einer nicht eingegangenen oder abgebrochenen Therapie gehört bei gerichtlich angeordneten Massnahmen deren Abbruch, der Vollzug einer aufgeschobenen Haftstrafe oder die Anordnung der Verwahrung. Urteilsfähige Straftäter haben die Möglichkeit zur Entscheidung, auch wenn es eine Wahl zwischen Skylla und Charybdis ist. Bei nicht Urteilsfähigen ist analog zum Erwachsenenschutzrecht vorzugehen.

Als «sittenwidrig» bezeichnet Herr Dr. Gmür die «Androhung von Repressalien wie Verwahrung, Verweigerung von Ausgängen, Disziplinarstrafen usw.». Würde dies in der Tat regelhaft im Sinne einer Androhung geschehen, hätte er Recht. Allerdings geht es wohl eher darum, dem Massnahmepatienten die Konsequenzen einer Therapieverweigerung deutlich zu machen. Diese liegen nicht im Ermessen der Therapeuten, sondern in der Hand der Justiz. Dennoch ist es nach Ansicht der Autoren nur fair, wenn man dem Straftäter erklärt, dass eine Therapieverweigerung nicht zur Besserung der Kriminalprognose beiträgt, was zur Anordnung einer Verwahrung führen kann. Dies mag subjektiv als Drohung erlebt werden, ist letztlich aber die Darlegung möglicher Konsequenzen und weist vom Procedere her Ähnlichkeiten mit der ärztlichen Aufklärung vor einer Operation auf. Auch dabei werden die Konsequenzen von Zustimmung bzw. Ablehnung verdeutlicht, ohne dass dies unbedingt einen Drohcharakter haben muss.

Wenn Mario Gmür fordert, das Arztgeheimnis im Rahmen von Massnahmebehandlungen uneingeschränkt zu gewährleisten, missachtet er, dass die forensische Therapie per se im Spannungsfeld zwischen den individuellen Interessen des Klienten und dem Sicherheitsbedürfnis der Allgemeinheit stattfindet. Sie muss beiden Aspekten Rechnung tragen. Es gibt nicht nur ethische Verpflichtungen gegenüber dem Patienten, sondern auch gegenüber potentiellen Opfern.

\section{Schlussbemerkungen}

Vor dem Hintergrund des vorab Gesagten überrascht nicht, dass die von Dr. Gmür gestellten Forderungen an die Forensische Psychiatrie fachlich jeder Grundlage entbehren oder bereits umgesetzt sind. Es ist selbstverständlich, dass psychotherapeutische Massnahmen das individualpräventive Potential der Strafe nicht verdrängen dürfen. Die Forensische Psychiatrie ist kein Ersatz zum Schuldstrafrecht. Überhaupt werden Tendenzen in Justiz und Politik, Straftäter unter dem Deckmantel einer vermeintlichen Behandlungsbedürftigkeit länger als im Strafrahmen vorgesehen in geschlossenen Settings führen zu können, von der Forensischen Psychiatrie ausgesprochen kritisch beobachtet. Dabei wurde auch von den Autoren vor einer unkritischen Erwartung an die Psychiatrie, kriminelle Neigungen als solche behandeln oder gar heilen zu können, und so vor einer Instrumentalisierung der Psychiatrie gewarnt [23].

Was ist zu tun? Bei sorgfältiger Prüfung der Evidenz ärztlichen Handelns im Rahmen der gesetzlichen und standespolitischen Regelungen kann die Forensische Psychiatrie einen wichtigen Beitrag zur angemessenen medizinischen und menschlichen Behandlung psychisch kranker Straftäter leisten und zum Schutz der Öffentlichkeit bzw. zu rechtsstaatlichen Verfahren und Urteilen beitragen. Die unsubstanziierten Ausführungen von Dr. Gmür haben mit einer solchen kritischen Prüfung nichts zu tun. Insofern bedauern die Autoren, dass Mario Gmür nunmehr auch in einer medizinischen Fachzeitschrift ein Podium geboten wurde, und hoffen, seine Polemik durch Argumente entkräftet zu haben. 


\section{Literatur}

1 Gmür M. Ethische Wegweiser für Prognosestellung und Psychotherapie im Strafrecht sowie im Straf- und Massnahmenvollzug. Schweiz Ärztezeitung. 2014; 95(26):1341-3.

2 Bleuler E. Das autistisch-undisziplinierte Denken in der Medizin und seine Überwindung. Berlin, Heidelberg: Springer Verlag 1919.

3 Hare RD. Manual for the Psychopathy Checklist - Revised. 2nd ed. Toronto: Multi Health Systems; 2003.

4 Harris A, Phenix A, Hanson RK, Thornton D. Static-99 Coding Rules Revised-2003. www.sgc.gc.ca; 2003.

5 Levenson JS. Reliability of sexually violent predator civil commitment criteria in Florida. Law Human Behav. 2004;28:357-68

6 Quesada SP, Calkins C, Jeglic EL. An examination of the interrater reliability between practitioners and researchers on the Static-99. Int J Offender Ther Comp Criminol. 2014; 58:1364-75.

7 Hare RD, Neumann CS, Mokros A. The PCL-R assessment of psychopathy: Development, properties, debates, and new directions. In: Patrick CJ, Ed. Handbook of psychopathy (2nd ed). New York: Guilford; in press.

8 Murrie DC, Boccaccini MT, Turner DB, Meeks M, Woods C, Tussey C. Rater (dis)agreement on risk assessment measures in sexually violent predator proceedings: Evidence of adversarial allegiance in forensic evaluation? Psychol Public Pol Law. 2009;15:19-53.

9 Grove WM, Meehl PE. Comparative Efficiency of Informa (Subjective, Impressionistic) and Formal (Mechanical, Algorithmic) Prediction Procedures: The Clinical-Statistical Controversy. Psychology, Public Policy and Law. 1996;2:293-323.

10 Hanson RK, Morton-Bourgon KE. The accuracy of recidivism risk assessments for sexual offenders: a meta-analysis of 118 prediction studies. Psychol Assess. 2009;21:1-21.

11 Webster CD, Haque Q, Hucker SJ. Violence Risk - Assessment and Management. 2. ed. Malden MA, USA: Wiley Blackwell; 2014.

12 Boetticher A, Kröber HL, Müller-Isberner R, Böhm KM, Müller-Metz R, Wolf T. Mindestanforderungen an Prognosegutachten. Neue Zeitschrift für Strafrecht. 2006;537-92.

13 Heer M, Habermeyer E. Art. 64 StGB. In: Niggli MA Wiprächtiger H, Hrsg. Basler Kommentar Strafrecht I. Basel: Helbing Lichtenhahn Verlag; 2013. S. 1496-528.
14 Olver ME, Nicholaichuk TP, Gu D, Wong SC. Sex offender treatment outcome, actuarial risk, and the aging sex offender in Canadian corrections: a long-term follow-up. Sex Abuse. 2013;25:396-422

15 Lipsey MW, Cullen FT. The effectiveness of correctional rehabilitation: a review of systematic reviews. Annual Review of Law and Social Science. 2007;3:297-320.

16 Stoffers J, Völlm BA, Rücker G, Timmer A, Huband N, Lieb K. Psychological therapies for people with borderline personality disorder. Cochrane Database of Systematic Reviews. 2012, DOI: 10.1002/14651858.CDo05652.pub2.

17 Bohus M, Stieglitz RD, Fiedler P, Hecht H, Herpertz S, Müller Isberner R, Berger M. Persönlichkeitsstörungen. In: Berger M, Hrsg. Psychische Erkrankungen. Klinik und Therapie. München Urban \& Fischer Verlag/Elsevier GmbH; 2014. S. 605-68.

18 Hare RD, Clark D, Grann M, Thornton D. Psychopathy and the Predictive Validity of the PCL-R: An International Perspective. Behav Sci Law. 2000;18:623-45.

19 Gibbon S, Duggan C, Stoffers J, Huband N, Völlm BA, Ferriter M, Lieb K. Psychological interventions for antisocial personality disorder. Cochrane Database of Systematic Reviews. 2010, DOI: 10.1002/14651858.CDoo7668.pub2.

20 Khalifa N, Duggan C, Stoffers J, Huband N, Völlm BA, Ferriter M, Lieb K. Pharmacological interventions for antisocial personality disorder. Cochrane Database of Systematic Reviews. 2010, DOI: 10.1002/14651858.CDoo7667.pub2

21 Dennis JA, Khan O, Ferriter M, Huband N, Powney MJ, Duggan C. Psychological interventions for adults who have sexually offended or are at risk of offending. Cochrane Database of Systematic Reviews. 2012, DOI: 10.1002/14651858.CDoo7507.pub2.

22 Marshall WL, Ward T, Mann RE, Moulden H, Fernandez YM, Serran G, Marshall LE. Working positively with sexual offenders maximizing the effectiveness of treatment. J Interpers Violence. 2005;20:1096-114.

23 Habermeyer E. Sicherungsverwahrung ist ein Thema für die Psychiatrie - Kontra. Psychiatrische Praxis. 2014;41:353-4. 